\title{
Editorial
}

\section{New technology in nutrition research and practice}

\begin{abstract}
Recent technology advancements are aiding the development of scientific discoveries and changing the methods by which we perform research. In order to gain full benefits for human health, it will be important to embrace these new technologies in nutrition research while also acknowledging their limitations. The present issue covers a range of technological approaches that impact on public health nutrition and molecular nutrition. The critical appraisal of these approaches in the context of nutrition research makes this issue a timely and pertinent addition to the scientific literature.
\end{abstract}

Nutrigenomics: Dietary assessment: Dietary biomarkers: Behavioural change: Technology

As technology becomes more and more advanced, it is timely to appraise its use in nutrition research. The Nutrition Society Summer Conference 2016 held in conjunction with the Irish Section of the Society set out to address this. From the outset, we wanted to break the classical concept that technology in nutrition is the use of a smart phone to aid research in some way. In the broader sense in science, it is much more far reaching than this, and in the design of this conference, we attempted to include as many of the advanced approaches as possible. This combined with the International Nutrition Student Research Championships, the Postgraduate Symposium, the Nutrition Society Medals and the Rank Prize lecture ensured that there was an excellent scientific programme with a broad range of topics.

A key challenge in nutrition research is obtaining reliable and accurate dietary data. Two symposia were dedicated to cover recent advancements in dietary assessment. Symposium 1 gave some great insights into the use and development of self-administered $24 \mathrm{~h}$ recalls using online platforms. Furthermore, the development of picture-based dietary assessment methods was highlighted. All of these approaches will no doubt continue to increase in the years to come and are set to make an important contribution to Nutrition Science. Complementary to the development of mobile technologies for assessment of dietary intake, there has been substantial research into the potential of dietary biomarkers as objective markers of intake. The second symposium covered this emerging field. Development of such markers is set to advance over the coming years, and combining these approaches with the mobile capturing approaches has the potential to significantly impact on how we assess dietary intake in the future.

Nutrigenomic technologies have been under development for many years, and now applications to nutrition research are making a significant impact on our understanding of the relationship between food and health. Examples of such technologies include metabolomics, transcriptomics and epigenetics. Of particular note is the growth in microbiome studies linking food intake to microbiome patterns and in turn to certain diseases. However, as with many of the other advanced technologies, care is needed when performing such studies and when analysing and interpreting the data. The present issue acknowledges this and will be a useful guide to those wishing to apply such technologies to their studies. Advanced phenotyping, including combining challenge tests with nutrigenomic techniques, offers the possibility of enhancing our understanding of the molecular mechanisms and will play an important role in the future development of biology-driven personalised nutrition.

Translation of knowledge gained in studies using new technologies into improvements in public health often requires new strategies for behavioural change. With this in mind, a symposium devoted to behavioural change strategies was well received by delegates and included topics surrounding decision-making in food choices, smart health and innovation and incentives to sustain healthy lifestyles. Incorporation and development of such approaches will become increasingly important as we attempt to translate findings from basic research.

As with any Nutrition Summer Conference, the Medal lectures were a highlight and offered the possibility for delegates to hear about exciting work from leaders in the broader field of nutrition. In the current issue, we have contributions that span a broad range of topics including but not limited to malnutrition, links between sugars and health and protective effects of $n-3$ PUFA. A key feature of the Nutrition Society meetings is the Postgraduate Symposium. This year, four students were selected to deliver oral presentations at this event. The range of topics was impressive with presentations covering the following areas: intermittent fasting, wholegrain recommendations, vitamin D deficiency and cholesterol metabolism.

Prior to the start of the conference, the International Nutrition Student Research Championships gave an excellent opportunity to listen to a range of topics from students elected by five European Nutrition Societies. The event supported by DSM gave the opportunity to early career scientists to present their work to an 
international audience. The range of topics covered was remarkable and included talks on personalised nutrition, glucose homeostasis and the FODMAP diet. The high calibre of talks and the enthusiasm surrounding the championships was infectious. Development of this event over the coming years will be important and offers the potential to interact with colleagues from a range of Nutrition Societies. In addition, a symposium on nutrient profiling was organised in conjunction with the Nestle Research Centre prior to the commencement of the main conference. Covered topics ranged from uses of nutrient profiling to address public health issues to regulatory purposes. This range of topics meant that the symposium in essence provided a one-stop shop for nutrient profiling.

As technology is having a significant impact on our everyday lives, it is also accelerating scientific discoveries and changing the methods by which we perform research. Embracing these new technologies and also acknowledging their limitations will be important as we move forward in this new era of research. The present issue covers a range of technological approaches that impact on public health nutrition and molecular nutrition. The critical appraisal of these approaches in the context of nutrition research makes this issue a timely and pertinent addition to the scientific literature.

Lorraine Brennan* and Breige McNulty UCD School of Agriculture and Food Science, UCD Institute of Food and Health, UCD, Belfield, Dublin 4,

Ireland

*Corresponding author: Professor L. Brennan, email lorraine.brennan@ucd.ie 